\title{
Impact of Protein-Polymer Interactions in the Antimicrobial Activity of Lysozyme/Poly(3,4-ethylenedioxythiophene) Biocapacitors
}

\author{
Jordi Triguero, ${ }^{[a]}$ David Zanuy, ${ }^{*[a]}$ and Carlos Alemán ${ }^{*[a, b]}$
}

\begin{abstract}
Biocapacitors constructed by combining lysozyme (LYZ) and poly(3,4-ethylenedioxythiophene) (PEDOT) retained the bactericidal activity of the protein when this was encapsulated within the polymeric matrix but lost the antimicrobial behaviour when the LYZ was adsorbed onto the polymer. In this work we use atomistic Molecular Dynamics simulations to examine the influence of protein $\cdots$ polymer interactions in the bactericidal activity of LYZcontaining biocapacitors. Results show that the anisotropic forces exerted by oxidized PEDOT chains on the adsorbed protein induce small structural changes that locally affect at the active centre, breaking the intra-residue interactions associated with the antibacterial mechanism. Conversely, isotropic polymer...protein interactions in biocapacitors with encapsulated LYZ do not affect the stability of the active centre. These observations suggest that the strong repulsive or attractive forces between $p$-doped polymer chains and the positively or negatively charged LYZ residues, respectively, are the only ones responsible for the protein activity.
\end{abstract}

\section{Introduction}

Biocapacitor is a recently coined term used for biodevices inspired by proteins to store and/or generate energy..$^{[1,2]}$ More specifically, single electrodes in which proteins are combined with synthetic materials, as for example conducting polymers (CPs), rechargeable devices able to store large amounts of electrical energy (supercapacitors) or to store the potential generated by a charge pump (enzymatic fuel cells), have been fabricated. ${ }^{[1-5]}$ The potential of biocapacitors in the biomedical field is enormous since the protein can help not only to improve the biocompatibility of the devices for in vivo implants, ${ }^{[2,4]}$ but also can be used to develop bioinspired sensing actuators for drug delivery/pumping systems. ${ }^{[5]}$ In recent studies we reported the preparation of electrodes for biocapacitors using chicken egg white lysozyme (LYZ), also known as muramidase, and poly(3,4ethylenedioxythiophene) (PEDOT). ${ }^{[2,4]}$

[a] J. Triguero, Dr. D. Zanuy, Prof. Dr. C. Alemán

Departament d'Enginyeria Química

Universitat Politècnica de Catalunya

EEBE, C/ Eduard Maristany, 10-14, Ed. 12, 08019, Barcelona, Spain

E-mail: david.zanuy@upc.edu and carlos.aleman@upc.edu

[b] Prof. Dr. C. Alemán

Barcelona Research Center for Multiscale Science and Engineering Universitat Politècnica de Catalunya

EEBE, C/ Eduard Maristany, 10-14, Ed. C, 08019, Barcelona, Spain

Supporting information for this article is given via a link at the end of the document
Although LYZ is mostly known for its hydrolase activity on the peptidoglycan of Gram-positive bacteria, recent studies have evidenced that this small protein of 129 residues $(14.4 \mathrm{kDa})$ also exhibits activity on Gram-negative bacteria. ${ }^{[6]}$ In fact, there are numerous reports on the usage of $L Y Z$ as antimicrobial agent in food processing ${ }^{[7]}$ and biomedical applications, as for example biosensing devices and therapeutic. ${ }^{[8]}$ This natural antibiotic exhibits an ellipsoidal shape in which ordered secondary structures ( $\sim 40 \% \alpha$-helix and $\sim 10 \% \beta$-sheet) coexist with a large amount of random coils and turns. ${ }^{[9]}$

Among conjugated polymers CPs, PEDOT (Scheme 1) is one of the most important because of its great environmental stability, electrical conductivity, electrochemical activity, thermoelectric behavior and high specific capacitance. ${ }^{[10]}$ Additionally, PEDOT offers two fundamental advantages as compared to unsubstituted polythiophene (PTh) or other PTh derivatives: (1) the fused dioxane ring blocks the $\beta$-position of the thiophene ring preventing from the formation of $\alpha-\beta$ linkages during the polymerization, which makes the resulting polymer well-defined from a regiochemical point of view; and (2) the fused dioxane ring and the electron-donating effects provided by the oxygen atoms cause the self-rigidification of polymer chains, resulting in a gain of aromaticity and in a reduction of the oxidative doping potential. ${ }^{[11]}$

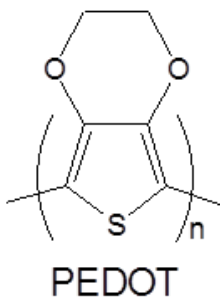

Scheme 1. Chemical structure of PEDOT

LYZ-containing PEDOT electrodes for biocapacitors were prepared conserving two approaches that differ in the strategy used to immobilize the protein. In the first one, the protein was simply adsorbed onto the surface of $\mathrm{CP}$ films. ${ }^{[4]}$ The resulting system, named PEDOT/LYZ, consisted on a biphasic system able to retain the electroactive and electrical properties of PEDOT but unable to protect against bacterial growth. In the second approach, LYZ was homogeneously distributed inside the PEDOT matrix, the resulting material being denoted $\mathrm{P}(\mathrm{EDOT}-\mathrm{LYZ})$ displaying excellent antibacterial activity. The latter was prepared by in situ polymerization using a medium containing both the protein, which acted as dopant agent, and the 3,4-ethylenedioxythiophene (EDOT) monomer. ${ }^{[2,4]}$ Thus, as 
CPs behave as dynamical systems able to exchange dopant ions with the medium, LYZ was slowly escaping from the polymer matrix to the culture medium while other ions (including other proteins) entered into it. ${ }^{[2,4]}$ After optimization of the polymerization conditions, P(EDOT-LYZ) hybrid electrodes exhibited higher electrochemical activity and biocompatibility than PEDOT. ${ }^{[4]}$ These results evidenced the potential of $\mathrm{P}(\mathrm{EDOT}-\mathrm{LYZ})$ as electrodes for electrochemical biocapacitors useful for in vivo implants.

In this work we use atomistic Molecular Dynamics (MD) simulations to explain the influence of protein...polymer interactions in the antimicrobial activity of LYZ-containing PEDOT electrodes. For this purpose, simulations have been conducted on two models that contain all the elements used for the fabrication PEDOT/LYZ and P(EDOT-LYZ) electrodes. Specifically MD simulations have been performed in absence and presence of electric fields, the latter mimicking the one originated by the voltage cell difference in biocapacitors. Interestingly, results indicate that the directional effects of the forces exerted by the CP on LYZ are responsible for the loss and retention of the biological activity in PEDOT/LYZ and $\mathrm{P}(\mathrm{EDOT}-\mathrm{LYZ})$, respectively, while unexpectedly the influence of the electric field in the loss of protein activity is negligible.

\section{Construction of PEDOT/LYZ and P(EDOT-LYZ) models and approach for the electric field}

The construction of both models started by placing one LYZ molecule in its crystal structure, 1LKS entry ${ }^{\left[{ }^{[b]}\right]}$ (Hen egg white lysozyme nitrate) from the Protein Data Bank (PDB), at the centre of a cubic simulation box $(a=190 \AA)$ fulfilled with 19508 previously equilibrated ( $1 \mathrm{~atm}$ and $298 \mathrm{~K}$ ) explicit water molecules. The charge of all potential titratable residues (6 Lys, $11 \mathrm{Arg}, 7 \mathrm{Asp}$ and $2 \mathrm{Glu}$ ) was fixed to the values corresponding to neutral $\mathrm{pH}$ (i.e. Asp and Glu side chains were negatively charged, while Lys and Arg side groups were represented in their positively charged forms). Then, eight $\mathrm{ClO}_{4}{ }^{-}$anions were added to the simulation box, close to positively charged residues to reach the neutrality. This system, hereafter denoted LYZ/water, was thermalized, equilibrated and relaxed using a three-step process, which can be summarized as follows: 1) $2 \times 10^{5}$ steps of energy minimization; 2) 2.1 ns of NVT MD at 298 $\mathrm{K}$; and 3) $35 \mathrm{~ns}$ of NPT MD at $298 \mathrm{~K}$ and 1.01325 bars. Figure $1 \mathrm{a}$, which displays the superposition of the $L Y Z$ as obtained from the last snapshot of the NPT MD simulation (blue) and in the crystal (red), indicates that the simulated protein maintains the structural characteristics observed in the solid state. Thus, the root mean square deviation (RMSD) between such two structures was of $2.162 \AA$ only.

Besides, an ultra-thin PEDOT film deposited over the surface of austenitic stainless steel, which was the substrate experimentally used to prepare both PEDOT/LYZ and P(EDOTLYZ) electrodes, ${ }^{[2,4]}$ was modelled using a previously described approach ${ }^{[12]}$ In brief, the steel substrate was represented using a $3 D$ organization of frozen iron atoms organized in the facecentred cubic (fcc) crystal lattice observed for steel (cell parameter: $a=3.65 \AA) .{ }^{[13]}$ Thus, as the metallic substrate acted as a simple anchoring base for PEDOT chains, the austenitic steel was represented as an iron lattice, its minor components being omitted from the model. Specifically, 13372 iron atoms were distributed in nine layers $(c=14.6 \AA)$ according to the (111) crystallographic plane, which corresponds to a surface of 98.55 $\times 100.35 \AA^{2}$. After this, 100 PEDOT chains with a number of EDOT units ranging between 68 and 138 were grown parallel to the crystallographic direction $c$ (z-axis) onto the iron substrate using a generation strategy that combines stochastic methods and MD simulations. ${ }^{[12]}$ This strategy was proved to reproduce the main nanofeatures (i.e. topography and morphology) experimentally observed at the surface of ultra-thin PEDOT films. The total charge of every EDOT unit was kept at +0.5 and, as determined experimentally in previous work. ${ }^{[14]}$ Therefore, one $\mathrm{ClO}_{4}^{-}$anion, which was the dopant agent experimentally used for the preparation of PEDOT/LYZ and P(EDOT-LYZ) electrodes, ${ }^{[2,4]}$ was introduced every two EDOT units to neutralize the system. $\mathrm{ClO}_{4}^{-}$ions were placed within the polymer matrix using an early strategy envisaged to identify free volume regions within any polymeric solid. ${ }^{[15]}$ Obviously, the density calculated for the PEDOT model, which contained 11000 EDOT units and 5500 perchlorate anions, $\rho=1.60 \mathrm{~g} / \mathrm{cm}^{3}$, fits the experimental density as determined by the flotation method, $\rho=$ $1.65 \mathrm{~g} / \mathrm{cm}^{3}{ }^{[14]}$ The resulting PEDOT model, which was equilibrated and relaxed using the protocol reported in our previous work, ${ }^{[12]}$ is displayed in Figure $1 \mathrm{~b}$.

PEDOT/LYZ and P(EDOT-LYZ) models were constructed placing the PEDOT model in orthorhombic cells of $98.55 \times$ $100.35 \times 559.0 \AA^{3}$ and $98.55 \times 100.35 \times 601.0 \AA^{3}$, respectively, and using the results from the LYZ/water simulation. Specifically, the PEDOT/LYZ model was built by transferring the LYZ protein, the eight $\mathrm{ClO}_{4}^{-}$anions and the water molecules from the first hydration shell (i.e. water molecules with the oxygen atom at a distance shorter than $2.5 \AA$ from any polar atom of the protein or the $\mathrm{ClO}_{4}{ }^{-}$anions), as obtained from the last snapshot of the LYZ/water simulation, onto the PEDOT surface. The remaining free space of the simulation box was filled with water molecules from a previously equilibrated simulated solvent box that reproduced the experimental density of this liquid. This PEDOT/LYZ model, which is depicted in Figure 1c, contained 13372 iron atoms, 11000 EDOT units, one LYZ molecule, 5508 $\mathrm{ClO}_{4}{ }^{-}$anions and 112102 water molecules (i.e. a total of 522286 explicit atoms). Finally, the whole system was relaxed and equilibrated using the protocol described in the Methods section.

For the construction of the P(EDOT-LYZ) model a four-step strategy was engineered to encapsulate the protein inside the CP matrix. First, a very efficient approach ${ }^{[15]}$ was used to identify all regions with unoccupied volume within the PEDOT film model (Figure 1b). After this, the largest unoccupied region was selected and filled with four spherical fictitious atoms of radius $R=10 \AA$ and well depth $\varepsilon=0.20 \mathrm{kcal} / \mathrm{mol}$. Third, the whole system, constituted by the PEDOT film model plus the four fictitious atoms was minimized and, subsequently, relaxed using a short NVT MD (5 ns). This step was repeated 15-times increasing slowly both the radius and well depth of fictitious atoms (i.e. $\Delta R=2 \AA$ and $\Delta \varepsilon=0.1 \mathrm{kcal} / \mathrm{mol}$ ) each time until reach 
the following parameters: $R=40 \AA$ and well depth $\varepsilon=0.35$ $\mathrm{kcal} / \mathrm{mol}$. Finally, the four fictitious atoms were replaced by the $\mathrm{LYZ}$, the eight $\mathrm{ClO}_{4}^{-}$anions and the water molecules from the first hydration shell as obtained from the last snapshot of the LYZ/water simulation. Finally, the P(EDOT-LYZ) model was completed by filling the free space of the simulation box with water molecules from a previously equilibrated simulated solvent box. This model, which was subjected to relaxation and equilibration as described in the Methods section, is displayed in Figure 1d and contained 13372 iron atoms, 11000 EDOT units, one LYZ molecule, $5508 \mathrm{ClO}_{4}^{-}$anions and 124996 water molecules (i.e. a total of 560968 explicit atoms). (a)

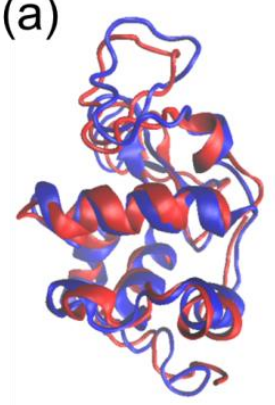

(c)

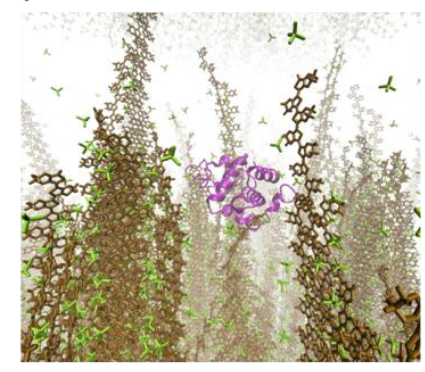

(d)

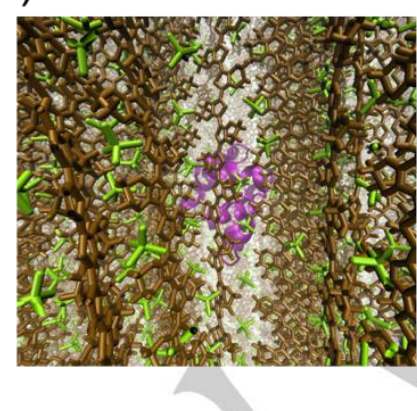

(b)
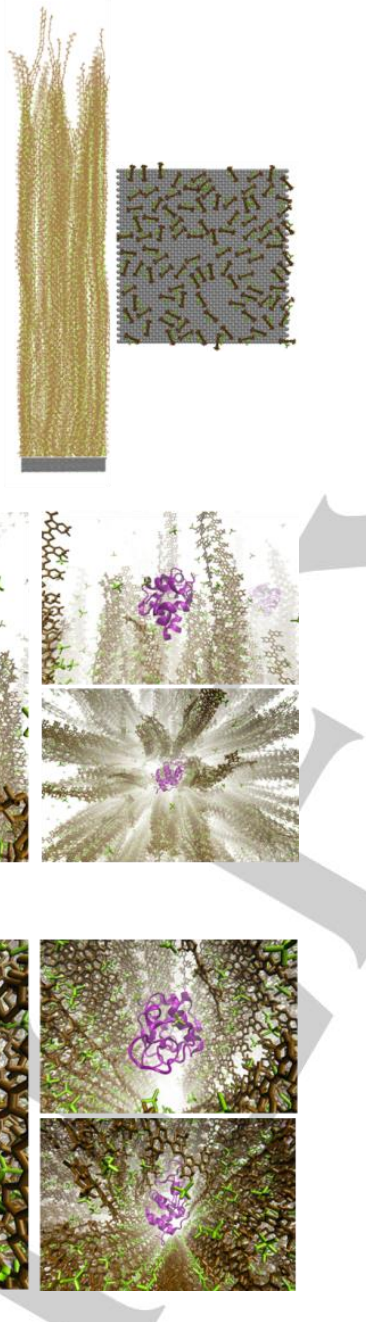

Figure 1. (a) Superposition of the LYZ in the crystal (1LKS entry in PDB; in red) and as obtained from the MD relaxation of the LYZ/water model (last snapshot from a 35 ns NPT MD simulation; in blue). (b) Lateral view (left) of the model constructed to mimic ultra-thin PEDOT films deposited onto stainless steel. The relative position of the PEDOT chains onto the iron substrate is also displayed (right). (c,d) Different views of the (c) PEDOT/LYZ and the (d) $P(E D O T-L Y Z)$ models illustrating the position of the protein molecule relative to the $\mathrm{CP}$ chains. The two models were constructed combining the results derived from the LYZ/water simulation and the PEDOT film model, as it is described in the text. The protein, PEDOT chains and $\mathrm{ClO}_{4}{ }^{-}$ anions are represented in purple, brown and yellowish green, respectively, while the iron substrate and the water molecules have been omitted for clarity.
The most direct approach for simulating an electric field consists of constructing a layer of explicit positive charges on one side of the simulation box and another layer of explicit negative charges on the opposite side of the simulation box (i.e. the system comprises two parallel layers with fixed charges separated by the bulk phase). Different charge concentrations in each layer can be introduced to create a realistic potential. However, the dual-layer approach considerably increases the size of the simulated systems and the computational cost. Thus, in order to avoid unrealistic border effects derived from artificially created layers, the bulk phase is required to be large enough. Although the dual-layer method might become more popular as computational power increases and simulations of larger systems become accessible, the constant electric field approach allows to avoid such undesirable effects. In this strategy, a uniform electric field, $\overrightarrow{E_{z}}$, is introduced throughout the entire simulated periodic cell. This gives rise to a force $q_{i} \overrightarrow{E_{z}}$ that applies to all charges $q_{i}$ in the simulation. The electric field is directed perpendicular to the box plane and must have a magnitude $\overrightarrow{E_{z}}=V / L_{z}$, where $L_{z}$ is the length of the simulation box in that direction. Thus, the value of the applied voltage $V$ is known a priori and the magnitude of the applied field depends only on the size of the simulation box $L_{z}$, with no need for a duallayer with an enlarged system. This approach, which has been employed in this work, was successfully used in many applications studying ion conduction, voltage-regulated water flux, and induced conformational changes of membrane proteins. ${ }^{[16]}$

\section{Results and Discussion}

\section{Structural analysis of the adsorbed and encapsulated protein}

Figures $2 \mathrm{a}$ and $3 \mathrm{a}$ display the temporal evolution of the RMSD for the protein in PEDOT/LYZ and P(EDOT-LYZ) models, respectively, which was calculated with respect to the initial conformation and considering all backbone and side chain nonhydrogen-atoms. In absence of electric field the RMSD of the protein exhibits small fluctuations in both models. Considering the last $20 \mathrm{~ns}$ of each trajectory, the average RMSD is $2.52 \pm 0.91$ and $2.04 \pm 0.08 \AA$ for PEDOT/LYZ and P(EDOT-LYZ), respectively, suggesting that the backbone remains structurally stable during the whole simulations while side chains experience some deviations. It is worth noting that the structural distortion is higher when protein is adsorbed onto the CP surface than when it is embedded inside the CP matrix.

Amazingly, the electric field does not cause relevant changes in the RMSD (Figures $2 \mathrm{a}$ and $3 \mathrm{a}$ ), independently of its strength and sense. Indeed, in all cases the RMSD is lower in presence than in absence of electric field. For example, the averaged RMSD considering the last 20 ns of the 60 ns production run for PEDOT/LYZ and P(EDOT-LYZ) is $2.01 \pm 0.13$ and $1.75 \pm 0.08 \AA$, respectively, when $\overrightarrow{E_{z}}=0.20 \mathrm{kcal} /\left(\mathrm{mol} \cdot \AA \cdot \mathrm{e}^{-}\right)$, which represents a reduction of around $15-20 \%$ with respect to the averages obtained in absence of electric field. This tendency is clearly maintained when the length the trajectories was 
increased to 250 ns (i.e. the averaged RMSD considering the last 200 ns of the 250 ns run for PEDOT/LYZ and P(EDOT-LYZ) with when $\overrightarrow{E_{z}}=0.20 \mathrm{kcal} /\left(\mathrm{mol} \cdot \AA \cdot \AA \cdot \mathrm{e}^{-}\right)$is $2.31 \pm 0.18$ and $1.65 \pm 0.14 \AA$, respectively), as is reflected in Figure $\mathrm{S} 1$.
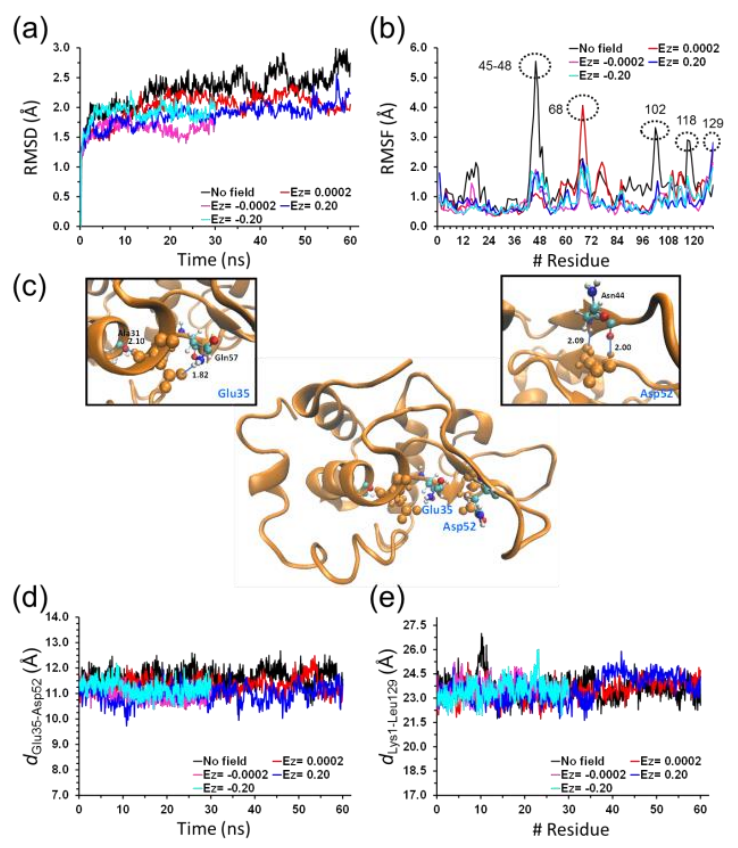

Figure 2. For PEDOT/LYZ simulations in absence and presence of electric field: (a) Temporal evolution of the RMSD, which was calculated considering all the protein non-hydrogen-atoms; (b) Residue based RMSF at the end of the simulation, which was calculated considering all the protein non-hydrogenatoms; (c) Details about the interactions formed by the Glu35 and Asp52 residues (represented by balls in light brown), which are responsible for the antimicrobial activity of LYZ. Magnification of the Glu35- and Asp52-containing regions is displayed (left and right, respectively). (d,e) Distances (in $\AA$ ) correspond to specific inter-residue interactions; and temporal evolution of the distance between the $\mathrm{C} \alpha$-atoms of (d) Glu35 and Asp52 ( $d_{\text {Glu35-Asp52), and (e) }}$ Lys1 and Leu129 ( $d_{\text {Lys1-Leu129) }}$.

To assess the location of the major distortions of the constituting residues, the root mean square fluctuations (RMSF) of all nonhydrogen atoms from the protein in PEDOT/LYZ and P(EDOTLYZ) was measured with respect to their positions in the crystal (Figures $2 \mathrm{~b}$ and $3 \mathrm{~b}$, respectively). According to the Phillips mechanism, ${ }^{[17]}$ Glu35 and Asp52 residues play an essential role in the antibacterial mechanism of LYZ. The protein damages bacterial cells by cleaving the $\beta(1 \rightarrow 4)$ glycosidic linkage between alternating units of $\mathrm{N}$-acetylmuramic acid and $\mathrm{N}$ acetylglucosamine, which are building blocks of bacterial cell walls. The terminal proton of Glu35 is transferred to the oxygen atom of the glucosidic bond between two neighbouring sugar residues, leading to the cleavage of the glucosidic bond and the formation of the carbenium ion. The positive charge of this carbenium ion is stabilized by the negative charge of Asp52 until a hydroxyl ion binds to the positive carbon atom and Glu35 is reprotonated. Another reaction mechanism, proposed by Koshland ${ }^{[18]}$ involves a glycosyl-LYZ intermediate in which the substrate is covalently bonded to Asp52. Vocadlo et al. ${ }^{[9]}$ concluded from electrospray-ionization mass-spectrometric studies that the catalytic mechanism of LYZ involves an intermediate complex in which one sugar ring is covalently bonded to Asp52. However, the crystallized structure of a trisaccharide bound to the active-site of LYZ did not present such covalent intermediate, while the presence of hydrogen bonds in the environment of Glu35 and Asp52 supported the Phillips mechanism. ${ }^{[20]}$ Inspection of the LYZ crystal structure ${ }^{[9 b]}$ shows that the carboxylate side group of Glu35 interacts with the following residues: Gln57 ( $\left.\mathrm{N}_{\mathrm{bb}} / 1.76 \AA\right)$, Val109 $\left(\mathrm{N}_{\mathrm{bb}} / 1.82 \AA\right)$ and Ala110 $\left(\mathrm{N}_{\mathrm{bb}} / 1.84 \AA\right)$, where $\mathrm{N}_{\mathrm{bb}}$ refers to the nitrogen atom from the backbone and the distance corresponds to the $\mathrm{N}-\mathrm{H} \cdots \mathrm{O}$ interaction. Besides, the carboxylate of the Asp52 residue interacts with $\operatorname{Arg} 45\left(\mathrm{~N}_{\mathrm{sc}} / 1.81 \AA\right.$; $\mathrm{N}_{\mathrm{sc}} / 2.10 \AA$; double salt bridge), Asn46 ( $\left.\mathrm{N}_{\mathrm{bb}} / 1.91 \AA\right)$, Thr47 $\left(\mathrm{N}_{\mathrm{bb}} / 2.09 \AA\right)$ and Asn59 $\left(\mathrm{N}_{\mathrm{sc}} / 1.82 \AA\right)$, where $\mathrm{N}_{\mathrm{sc}}$ denotes the nitrogen atom from the side chain. In addition, the backbone $\mathrm{N}-\mathrm{H}$ and $\mathrm{C}=\mathrm{O}$ groups of Glu35 and Asp52 also participate in specific hydrogen bonding

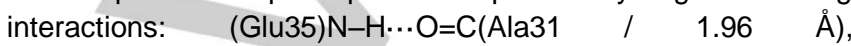

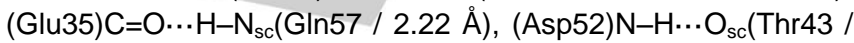
$2.04 \AA)$ and $\left(\right.$ Asp52) $\mathrm{C}=\mathrm{O} \cdots \mathrm{H}-\mathrm{O}_{\mathrm{sc}}(\mathrm{Thr} 43 / 1.62 \AA)$. These hydrogen bonds and salt bridges, which are responsible for the antimicrobial activity of $L Y Z$ are depicted in Figure $S 2$.

In absence of electric field, the RMSF of Glu35 and Asp52 in PEDOT/LYZ are relatively low: 0.89 and $1.65 \AA$, respectively (Figure $2 b$ ). However, in this system PEDOT...LYZ interactions cause drastic distortions in surrounding regions that are crucial for the activity of the protein. Figure 2c shows that Glu35 only preserves the side chain $\cdots$ backbone interaction with Gln57 (1.82 $\AA$ ) and the backbone $\cdots$ backbone hydrogen bond with Ala31 $(2.10 \AA)$, which is associated to the $\alpha$-helix secondary structure. This reduction is even more drastic for Asp52 since all the interactions mentioned above disappear upon adsorption onto PEDOT surface. Instead two new $\mathrm{N}-\mathrm{H} \cdots \mathrm{O}$ interactions with the backbone of Asn44 are formed due to the apparition of a $\beta$-sheet. Figure $2 \mathrm{~b}$ shows the very high RMSF values of $\operatorname{Arg} 45$ (3.72 $\AA$ ), Asn46 (5.55 $\AA$ ) and Asp47 (4.61 $\AA$ ), which explains the drastic change in the interaction pattern of the active site with respect to the crystal.

Independently of its strength and sign, the RMSFs decreases in presence of $\overrightarrow{E_{z}}$ (Figure $2 b$ ), which is consistent with the RMSD behaviour. In spite of this, the greater distortions and, by extension, the lost inter-residue interactions involving Glu35 and Asp52 are the same in absence and presence of electric

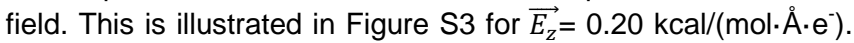
Overall, MD simulations on PEDOT/LYZ indicate that the loss of antimicrobial activity is due to the distortions induced by the PEDOT...LYZ interactions formed upon adsorption rather than to the applied electric field. Although the alterations caused by these interactions are not very drastic, as is reflected by the superposition of the crystal structure and the last snapshot from the simulation (Figure S4), they affect the side groups of key polar residues, altering significantly the interaction pattern with respect to the crystal (Figures S2 and 2c).

Regarding to $\mathrm{P}(\mathrm{EDOT}-\mathrm{LYZ})$, many of the interactions involving Glu35 and Asp52 remain practically unaltered, as it is shown in Figure 3c. More specifically, the Glu35 maintains the 
side chain $\cdots$ backbone interactions with Val109 (1.82 Å) and Ala110 (2.35 $\AA)$ and the backbone...backbone interaction with Ala31 (1.95 ̊), losing the two interactions with Gln57. The Asp52 preserves the side chain $\cdots$ backbone interaction with Asn46 (2.24 $\AA$ ), the side chain...side chain with Asn59 (this becomes stronger, involving the two oxygen atoms of the carboxylate moiety: 2.41 and $2.20 \AA$ ), and one of the backbone $\cdots$ side chain interactions with Thr43 (1.87 ̊). Thus, Asp52 loses the interactions with Arg45 and Thr47, even though new interactions with Asn44 (Figure 3c), similar to those found for PEDOT/LYZ, are detected. Moreover, many of the interactions found in the crystallized active centre are also detected in P(EDOT-LYZ) when the $\overrightarrow{E_{z}}$ is applied, independently of the strength and sign. This is illustrated in Figure S5 for $\overrightarrow{E_{z}}=$ $0.20 \mathrm{kcal} /\left(\mathrm{mol} \cdot \AA \cdot \AA \cdot \mathrm{e}^{-}\right)$, which shows the interactions of Glu35 with Ala31 (1.84 $\AA)$, Val109 (2.03 $\AA$ ) and Ala110 (2.04 $\AA$ ) and of Asp52 with Thr43 (1.83 $\AA$ ), Asn44 (2.02 and $2.04 \AA)$, Asn46 $(2.49 \AA)$ and Thr47 (1.55 and $1.90 \AA)$. (a)

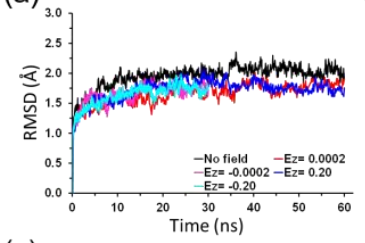

(c)

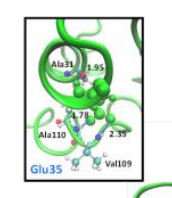

(b)

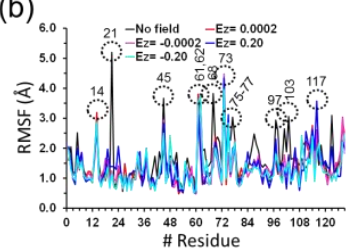

both simulations in absence of electric field and applying $\overrightarrow{E_{z}}=$ $0.20 \mathrm{kcal} /\left(\mathrm{mol} \cdot \AA \cdot \mathrm{e}^{-}\right)$being considered for the latter two. Unfortunately, from these results it is not possible to ascertain which residue, Glu35 or Asp52, plays the most important role in the antimicrobial activity of LYZ since almost all interactions are lost in PEDOT/LYZ.

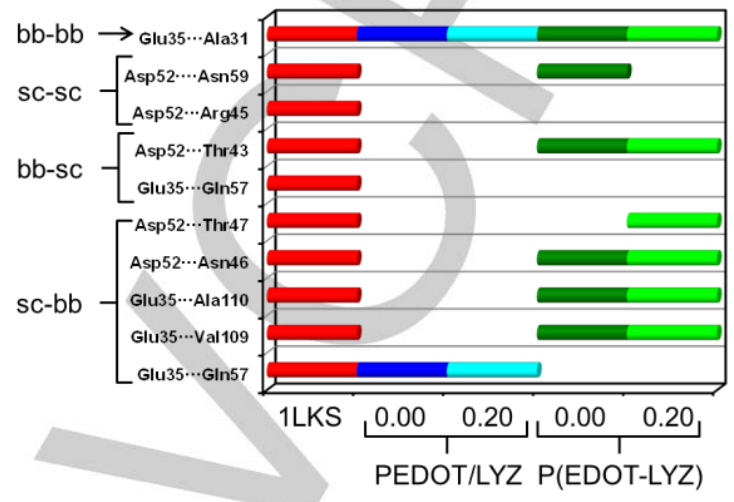

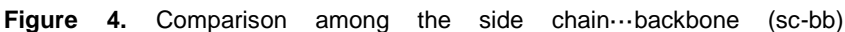
backbone-..side chain (bb-sc), side chain...side chain (sc-sc) and backbone $\cdots$ backbone (bb-bb) interactions involving Glu35 and Asp52 in 1LKS (crystal structure), PEDOT/LYZ and P(EDOT-LYZ). Observed interactions are represented by coloured bars. Results from simulations in absence of electric field and applying $\overrightarrow{E_{z}}=0.20 \mathrm{kcal} /\left(\mathrm{mol} \cdot \AA \cdot \mathrm{e}^{-}\right)$are considered for the two composites with PEDOT. (d)

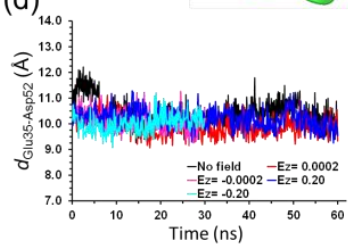

(e)

Figure 3. For $P(E D O T-L Y Z)$ simulations in absence and presence of electric field: (a) Temporal evolution of the RMSD, which was calculated considering all the protein non-hydrogen-atoms; (b) Residue based RMSF at the end of the simulation, which was calculated considering all the protein non-hydrogenatoms; (c) Details about the interactions formed by the Glu35 and Asp52 residues (represented by balls in green), which are responsible for the antimicrobial activity of LYZ. Magnification of the Glu35- and Asp52-containing regions is displayed (left and right, respectively). (d,e) Distances (in $\AA$ ) correspond to specific inter-residue interactions; and temporal evolution of the distance between the $\mathrm{C} \alpha$-atoms of (d) Glu35 and Asp52 ( $d_{\mathrm{Glu35-Asp52}}$ ), and (e) Lys1 and Leu129 ( $d_{\text {Lys1-Leu129). }}$.

Figure 4 summarizes the comparison among the side chain...backbone, backbone...side chain, side chain $\cdots$ side chain and backbone-..backbone interactions involving Glu35 and Asp52 in 1LKS (crystal), PEDOT/LYZ and P(EDOT-LYZ),

Overall, results indicate that the anisotropy of PEDOT $\cdots L Y Z$ interactions in PEDOT/LYZ are responsible for the loss of antimicrobial activity experimentally observed. ${ }^{[2]}$ Although such anisotropic interactions does not cause drastic changes in the conformation of LYZ, as is reflected by the RMSD and RMSF profiles displayed in Figures $2 a$ and $2 b$, respectively, they are large enough to affect the local interactions that preserves the stability of the active centre. This is clearly evidenced in Figure $2 \mathrm{~d}$, which represents the variation of the distance between the

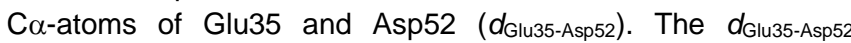
values averaged over the last $20 \mathrm{~ns}$ of each simulation are $11.69 \pm 0.36, \quad 11.00 \pm 0.35, \quad 11.38 \pm 0.38, \quad 10.84 \pm 0.22$, and $11.09 \pm 0.47 \AA$ for $\overrightarrow{E_{z}}=0.00,0.000 .2,0.20,-0.0020$ and -0.020 $\mathrm{kcal} /\left(\mathrm{mol} \cdot \AA \cdot \mathrm{e}^{-}\right)$, respectively. These values are approximately $15 \%$ larger than the one observed in the crystallized structure (i.e. $9.66 \AA$ ). Figure $2 e$ depicts the temporal evolution of the distance between Lys1 and Leu129 ( $d_{\text {Lys1-Leu129), which can be }}$ interpreted as the breathing movements of the LYZ protein during the trajectory. As it can be seen, fluctuations are very small, suggesting that the polymer stretches the part of the protein in contact with the surface. This avoids the small movements associated with the thermal effects and cause local distortions. The average $d_{\text {Lys } 1 \text {-Leu129 }}$ values (last 20 ns of each simulation), which are $23.55 \pm 0.52,23.89 \pm 0.44,24.58 \pm 0.51$, $23.61 \pm 0.41$, and $23.59 \pm 0.64 \AA$ for $\overrightarrow{E_{z}}=0.00,0.0002,0.20$, 0.0002 and $-0.20 \mathrm{kcal} /\left(\mathrm{mol} \cdot \AA \cdot \mathrm{e}^{-}\right)$, respectively, are greater than the crystallographic value (i.e. $21.07 \AA)$. This overestimation $(\sim 13 \%)$ is fully consistent with the features discussed above.

A completely different situation is found for $P(E D O T-L Y Z)$, in which PEDOT....LYZ interactions are much more isotropic. In 
this case, the protein is kept enclosed in a cavity, which acts as a mold. The temporal evolution of $d_{\text {Glu35-Asp52 }}$ (Figure $3 d$ ) indicates values very close the crystallographic one, even when the electric field is applied. Specifically, the average $d_{\mathrm{Glu35-Asp52}}$ values (last $20 \mathrm{~ns}$ ) of each simulation are $10.31 \pm 0.40,9.87 \pm 0.34$ $10.07 \pm 0.39,10.11 \pm 0.39$, and $11.07 \pm 0.34 \AA$, or $\overrightarrow{E_{z}}=0.00,0.002$, $0.20,-0.002$ and $-0.20 \mathrm{kcal} /\left(\mathrm{mol} \cdot \AA \cdot \mathrm{e}^{-}\right)$, respectively, which differ by less than $6.7 \%$ with respect to the crystallographic value. On the other hand, this cage-like effect observed for P(EDOT-LYZ) does not affect the breathing movement, fundamentally associated with the thermal energy, as is displayed in Figure 3e. Thus, $d_{\text {Lys1-Leu129 }}$ experiences occasional deviations, recovering after a few ns values close to the average value. This behaviour should be attributed to the fact that cavity that contains the protein is large enough to prevent compressing affects but small enough to avoid structural distortions.

These conclusions are supported by relevant structural properties such as $R_{g}$ and $R_{H}$, which have been calculated to be 14.7 $\AA$ and $19.1 \AA$, respectively, for the crystallized protein. The values averaged from PEDOT/LYZ and P(EDOT-LYZ) simulations in absence of electric field are $\left[R_{g}=15.2 \pm 0.1 \AA\right.$; $R_{H}=$ $19.9 \pm 0.2 \AA]$ and $\left[R_{g}=15.1 \pm 0.1 \AA ; R_{H}=19.8 \pm 0.2 \AA\right]$, respectively. These values represent an increment with respect to the crystal of $3 \%-4 \%$ only, supporting that the CP does not induce severe structural distortions in the protein. Moreover, the changes caused by the application of the electric field are very small, independently of the strength and sign (Table S1).

Analysis of the secondary motifs in the crystal indicates that the content of $\beta$-strands and helices amounts to $\beta S=3.1 \%$ and $\mathrm{H}=37.2 \%$, whereas loops- and turns-containing unfolded regions reach the highest population $L+T=59.7 \%$. Figure 5 represents the temporal evolution and average value of the population of $\beta S, H$ and $L+T$ motifs for PEDOT/LYZ and $P(E D O T-L Y Z)$ in absence of electric field and applying $\overrightarrow{E_{Z}}=0.20$ $\mathrm{kcal} /\left(\mathrm{mol} \cdot \AA^{-} \cdot \mathrm{e}^{-}\right)$, which has been chosen to illustrate the effect of the electric field (i.e. trajectories with $\overrightarrow{E_{z}}=0.002,-0.002$ and -0.20 $\mathrm{kcal} /\left(\mathrm{mol} \cdot \AA \cdot \mathrm{A}^{-}\right)$provide similar results). Interestingly, in absence of electric field both the adsorption and the encapsulation of LYZ (Figures $5 \mathrm{a}$ and $5 \mathrm{~b}$, respectively) result in a significant increment of $\beta$-strands, which is possible due to a reduction in the population of unstructured regions, while helices remain approximately at the same value (i.e. differences were $1.0-1.2 \%$ only). However, these tendencies are more pronounced for PEDOT/LYZ $(\beta S=11.0 \% \pm 0.9 \%$ and $L+T=50.6 \% \pm 2.3 \%)$ than for $\mathrm{P}(\mathrm{EDOT} / \mathrm{LYZ}) \quad(\beta \mathrm{S}=8.1 \% \pm 0.9 \%$ and $\mathrm{L}+\mathrm{T}=53.7 \% \pm 2.2 \%)$. Application of $\overrightarrow{E_{z}}=0.20 \mathrm{kcal} /\left(\mathrm{mol} \cdot \AA \cdot \mathrm{e}^{-}\right)$to the system with the adsorbed protein is in detriment of the $\beta S$ and $H$ populations (Figure 5c), which increase with respect to the crystal, causing a reduction in the percentage of $L+T$ that is higher than that obtained in absence of electric field. Conversely, the electric field does not produce any significant change in the distribution of secondary motifs of P(EDOT-LYZ) (Figure $5 \mathrm{~d}$ ), the populations of secondary motifs being similar to those achieved in absence of electric field.

On the basis of these results and the previously reported experimental observations, ${ }^{[2]}$ the mechanisms that regulates the antimicrobial activity of $\mathrm{P}(\mathrm{EDOT}-\mathrm{LYZ})$ is proposed to be based on the dynamics of the PEDOT chains. Thus, CPs are dynamic systems in which dopant ions are continuously exchanged with the medium (i.e. access of ions into the polymer matrix and escape of ions from the polymer matrix). This exchange is accompanied by relatively slow structural re-arrangements and local relaxations of the polymer chains, which probably facilitate the slow diffusion and subsequent release the LYZ molecules towards the medium.
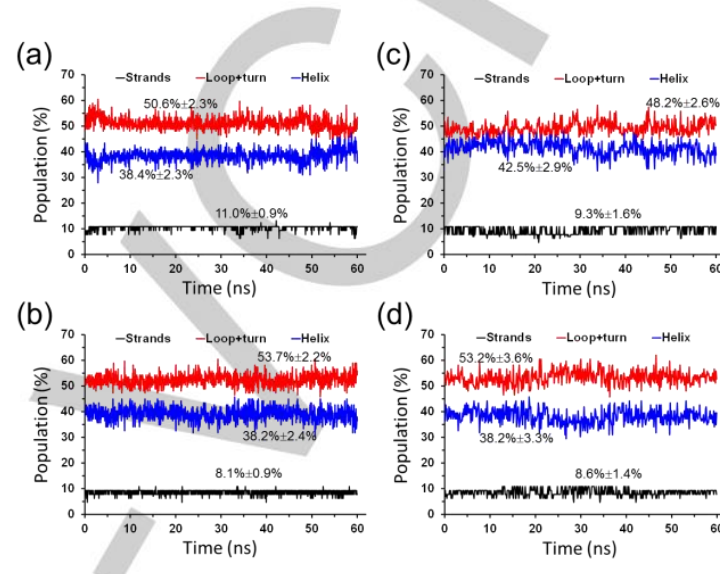

(d)

Figure 5. Temporal evolution of the population of $\beta$-strands $(\beta S)$, helices $(H)$ and loops and turns $(\mathrm{L}+\mathrm{T})$ as derived from $(\mathrm{a}, \mathrm{c})$ PEDOT/LYZ and $(\mathrm{b}, \mathrm{d})$ $\mathrm{P}(\mathrm{EDOT} / \mathrm{LYZ}) \mathrm{MD}$ trajectories in $(\mathrm{a}, \mathrm{b})$ absence of electric field and $(\mathrm{b}, \mathrm{d})$

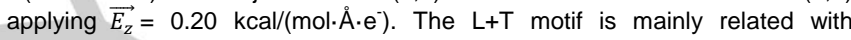
unstructured regions of the protein. Average values with the corresponding standard deviations, which were calculated considering the whole trajectories, are also displayed

\section{PEDOT $\cdots$ LYZ interactions}

The interactions between the LYZ protein and PEDOT chains have been analysed by calculating the radial distribution functions (RDFs) between pairs of atoms. Figure 6a, which represents the RDFs calculated between all atoms of $L Y Z$ and all atoms of PEDOT chains atom (RDF $F_{\text {LYZPEDOT), reflect the }}$ presence of van der Waals contacts in both PEDOT/LYZ and $\mathrm{P}($ EDOT-LYZ). In both cases the profiles start at $r \approx 2.3 \AA$, which is shorter to the sum of the van der Waals radii of hydrogen atoms, and increasing with $r$. This feature indicates the existence of non-specific electrostatic and van der Waals PEDOT...LYZ interactions. As it is expected, the growing tendency is much more pronounced for the protein embedded into the polymer matrix than for the protein adsorbed onto the surface of PEDOT.

Specific $\mathrm{N}-\mathrm{H} \cdots \mathrm{O}, \quad \mathrm{N}-\mathrm{H} \cdots \mathrm{S}, \mathrm{O}-\mathrm{H} \cdots \quad \mathrm{O}$ and $\mathrm{O}-\mathrm{H} \cdots \mathrm{O}$ hydrogen bonding interactions were examined through the corresponding LYZ(N or O) $\cdots(\mathrm{O}$ or S)PEDOT pairs. Obviously, the $\mathrm{O}$ atoms belonging to the backbone $\mathrm{C}=\mathrm{O}$ from $\mathrm{LYZ}$ were excluded from the analysis since they cannot act as hydrogen bond donor. Figures $6 b$ and $6 c$ display the RDFs $L Y Z(N)-(0)$ PEDOT

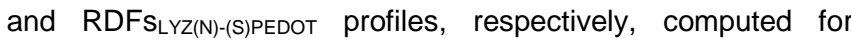
PEDOT/LYZ and P(EDOT-LYZ). A peak centred at $r \approx 4.6 \AA$ is obtained for the RDF $\mathrm{LYZ}_{\mathrm{N}(\mathrm{N})-(\mathrm{O}) \text { PEDOT }}$ of the two systems, even though this is significantly higher for PEDOT/LYZ than for $\mathrm{P}$ (EDOT-LYZ). The opposite tendency is shown for the first peak of the RDF $\mathrm{LYZ}_{\mathrm{N}(\mathrm{N})-(\mathrm{S}) \text { PEDOT }}$ at $r \approx 3.8 \AA$. These features confirm the 
existence of $\mathrm{N}-\mathrm{H} \cdots \mathrm{O}$ and $\mathrm{N}-\mathrm{H} \cdots \mathrm{S}$ hydrogen bonds in both the adsorbed and encapsulated systems. However, the former hydrogen bonds are more abundant in PEDOT/LYZ than in $\mathrm{P}$ (EDOT-LYZ), while the latter interaction is more abundant in $\mathrm{P}$ (EDOT-LYZ) than in PEDOT/LYZ. Interestingly, $\mathrm{N}-\mathrm{H} \cdots \mathrm{S}$ hydrogen bonds are stronger than $\mathrm{N}-\mathrm{H} \cdots \mathrm{O}$ ones, as is reflected by the $r$ values of the first peaks. This has been attributed to the flexibility of the dioxane ring, which is used to accommodate the protein onto the surface or inside the cavity of the CP film. Thus, the maximization of non-specific electrostatic interactions is in detriment of the strength of $\mathrm{N}-\mathrm{H} \cdots \mathrm{O}$ hydrogen bonds. In contrast, the strength of $\mathrm{N}-\mathrm{H} \cdots \mathrm{S}$ hydrogen bonds is imposed by the rigidity of the thiophene rings.

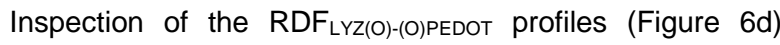
reveals that the characteristics of $\mathrm{O}-\mathrm{H} \cdots \mathrm{O}$ hydrogen bonds are similar to those discussed for the $\mathrm{N}-\mathrm{H} \cdots \mathrm{O}$ ones. Finally, $\mathrm{RDF}_{\mathrm{LYZ}(\mathrm{O}) \text {-(S)PEDOT }}$ profiles (not shown, respectively) evidence that $\mathrm{O}-\mathrm{H} \cdots \mathrm{S}$ interactions are practically negligible, no well-defined peak is detected. In general, results displayed in Figure 6 indicate that the electric field has a minor role in the population and strength of hydrogen bonds, causing only small changes.

\section{Perchlorate anions: Interactions and diffusion}

Finally, the strength and abundance of $\mathrm{ClO}_{4}{ }^{-} \ldots$ PEDOT and $\mathrm{ClO}_{4}^{-} \ldots \mathrm{LYZ}$ interactions were analysed by comparing the RDFs involving the following pairs of atoms: 1) the chlorine atom of $\mathrm{ClO}_{4}{ }^{-}$and the sulphur atoms of PEDOT chains (RDF ${ }_{\mathrm{Cl} \text {-(S)PEDOT); }}$ 2) the chlorine atom of $\mathrm{ClO}_{4}^{-}$and the backbone $\alpha$-carbon atoms

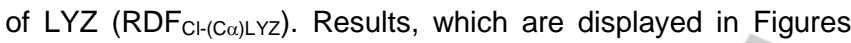
$\mathrm{S} 6 \mathrm{a}-\mathrm{b}$, indicate that the peaks are much higher for $\mathrm{RDF}_{\mathrm{CI}-(\mathrm{S}) \mathrm{PEDOT}}$

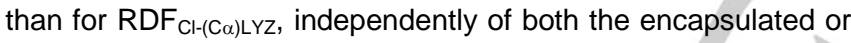
adsorbed configuration of the protein and the presence or absence of electric field. This feature is consistent with the fact that $\mathrm{ClO}_{4}^{-}$ions remain close to the PEDOT chains and only a few anions interact directly with the protein. Deeper analyses evidenced that such few $\mathrm{ClO}_{4}^{-} \ldots \mathrm{LYZ}$ interactions occur with the nitrogen atoms of Arg and Lys charged residues, as is shown by the RDFs calculated considering pairs involving the chlorine atom of $\mathrm{ClO}_{4}^{-}$and the nitrogen at the side chains of such residues $\left(\mathrm{RDF}_{\mathrm{Cl}-(\mathrm{Nsc}) \mathrm{LYZ}}\right.$ in Figure $\left.\mathrm{S} 6 \mathrm{c}\right)$. Moreover, these strong electrostatic interactions are more abundant in $\mathrm{P}$ (EDOT-LYZ) than in PEDOT/LYZ, as expected because of the encapsulation of the protein in $\mathrm{CP}$ matrix.

The diffusion coefficients $(D)$ of the $\mathrm{ClO}_{4}^{-}$ions were determined using the Einstein relation once the diffusive regime was reached. For this purpose, the mean square displacement as a function of time of $\mathrm{ClO}_{4}^{-}$ions were calculated. The values of $D$ calculated for PEDOT/LYZ and P(EDOT-LYZ) are represented in Figure 7 as a function of $\overrightarrow{E_{z}}$. In absence of electric field, the values of $D$ were similar for the two systems. Analysis of their three components (i.e. $D_{\mathrm{x}}, D_{\mathrm{y}}$ and $D_{z}$ ) indicates that $D_{z}\left(\sim 72 \cdot 10^{-5} \mathrm{~cm} / \mathrm{s}\right)$ is larger than $D_{\mathrm{x}}$ and $D_{\mathrm{y}}$, which are very similar $\left(\sim 47 \cdot 10^{-5} \mathrm{~cm} / \mathrm{s}\right)$. This feature indicates that, as it was expected, the diffusion of $\mathrm{ClO}_{4}^{-}$anions is higher in the direction perpendicular to the steel substrate than in the parallel one. The anisotropy in the diffusive behaviour of the dopant anions is guided by the orientation of the positively charged PEDOT chains, which grow perpendicular onto the iron substrate.

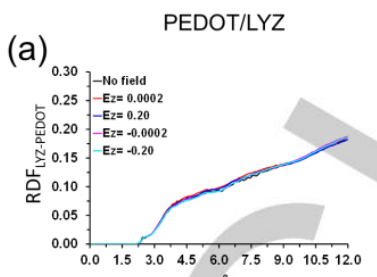

(b)

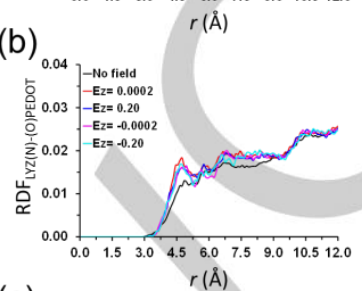

(c)

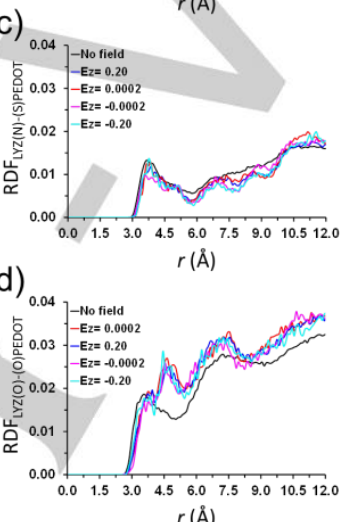

$r(\AA)$

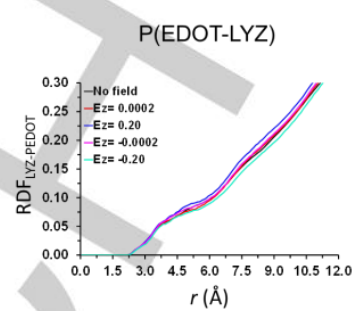

$r(\AA)$

$r(\AA)$

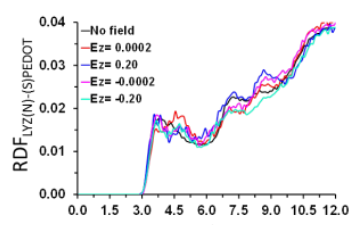

$r(\AA)$

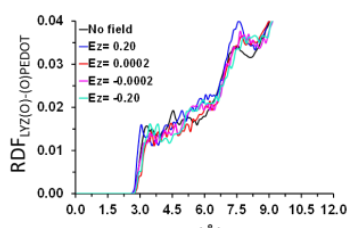

$r(\AA)$

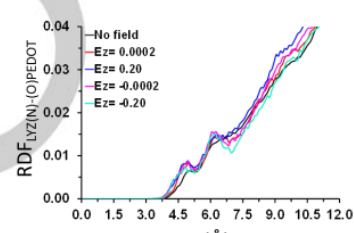

Figure 6. Radial distribution functions (RDF) for the following pairs of atoms: a) all atoms of LYZ and all atoms of PEDOT chains atom (RDF LYZ-PEDOT) to identify non-specific electrostatic and van der Waals interactions; b) $\mathrm{N}(-\mathrm{H})$ atoms of $L Y Z$ and $\mathrm{O}$ atoms of PEDOT (RDF $\left.\mathrm{RYZ}_{(\mathrm{N})-(\mathrm{O}) \text { PEDOT }}\right)$ to identify $\mathrm{N}-\mathrm{H} \cdots \mathrm{O}$

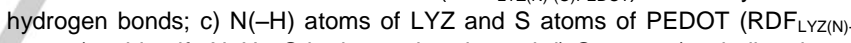
(S)PEDOT) to identify $\mathrm{N}-\mathrm{H} \cdots \mathrm{S}$ hydrogen bonds; and d) $\mathrm{O}$ atoms (excluding those belonging to the $\mathrm{C}=\mathrm{O}$ groups of the backbone) of $\mathrm{LYZ}$ and $\mathrm{O}$ atoms of PEDOT

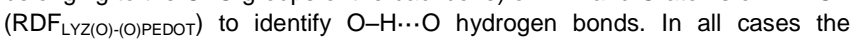
profiles calculated for PEDOT/LYZ and P(EDOT-LYZ) are displayed the left and right, respectively.

Application of $\overrightarrow{E_{z}}$.results in an enhancement of the diffusion coefficient, this effect being considerably more pronounced in the direction of the applied electric field than in the perpendicular direction. Moreover, such tendency increases with the strength of $\overrightarrow{E_{z}}$, especially for PEDOT/LYZ, while it is independent of sign of the field. Accordingly, the Arg and Lys residues of the protein immersed in the cavity inside the polymer matrix, remarkably shield the effects of the electric field in the diffusion of the dopant anions. Thus, the electric field is not high enough to disrupt the strong electrostatic interactions between the positive charged side groups and the negatively charged counter-anions. This feature is consistent with the fact that $\mathrm{RDF}_{\mathrm{Cl}-(\mathrm{Nsc}) \mathrm{LYZ}}$ is higher for P(EDOT-LYZ) than for PEDOT/LYZ (Figure S6c). 


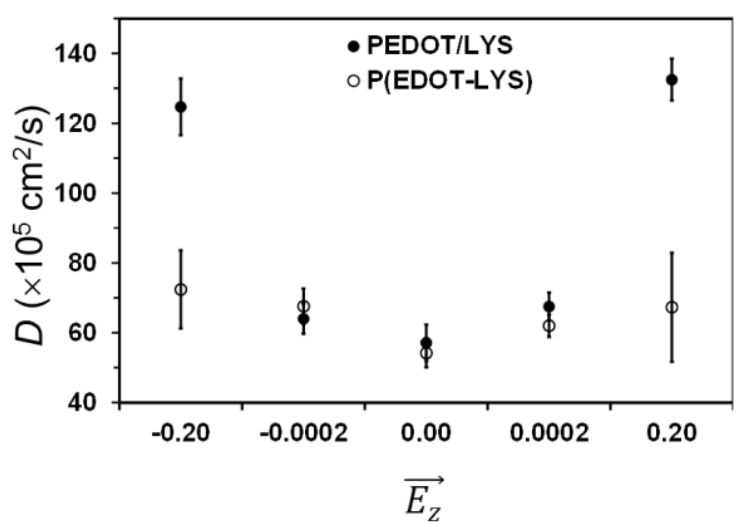

Figure 7. Diffusion coefficients of $\mathrm{ClO}_{4}^{-}$anions in $\mathrm{PEDOT} / \mathrm{LYZ}$ and $P(E D O T-L Y Z)$ in absence of electric field and applying selected external

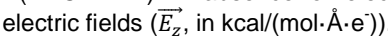

\section{Conclusions}

Our MD study gives support to the experimental observation that biocapacitors in which the LYZ is adsorbed onto the surface of PEDOT films do not present antimicrobial activity. Our results show that oxidized PEDOT chains, which grow onto a steel substrate and exhibit a total charge of +0.5 per EDOT repeat unit, interact with the numerous charged residues of the protein $(20.1 \%)$. These interactions produce small structural alterations at the protein, affecting the residues at the active centre and breaking the key interactions for the bactericidal activity of LYZ. Unexpectedly, this behaviour is independent of the presence of electric field, even when this is strong. Therefore, the loss of biological activity has been exclusively attributed to the anisotropy of the PEDOT ...LYZ electrostatic interactions, which is a phenomenon induced by the alignment of CP chains in the direction perpendicular to the metallic substrate. Conversely, biocapacitors prepared by encapsulating the protein inside the PEDOT matrix retain the most important inter-residue interactions at the $L Y Z$ active centre, preserving the antimicrobial activity. In this case, the anisotropic effects are minimized since the surrounding environment exerts forces of similar strength around the whole protein. Consequently, the formed electrostatic PEDOT...LYZ interactions are isotropic or quasi-isotropic and structural distortions are minimized.

The great advantage of the MD simulation technique is the wealth of structural information provided with atomistic detail. Although, this technique has a severe limitation in its high computational cost, which is particularly evident in simulations conducted in presence of electric field, realizable results can be obtained by exploring conditions adapted to drastically accelerate the investigated physical phenomena. For example, the length of the trajectories conducted using $\overrightarrow{E_{z}}=0.20$ and -0.20 $\mathrm{kcal} /\left(\mathrm{mol} \cdot \AA \cdot \mathrm{e}^{-}\right)$is enough to conclude unmistakably that the bactericidal activity of PEDOT/LYZ and P(EDOT-LYZ) biocapacitors is independent of the electric field applied or supplied during charge-discharge processes.

\section{Supporting Information Summary}

Experimental methods; radius of gyration and hydrodynamic radius in absence and presence of electric field; temporal evolution of the RMSD for PEDOT/LYZ and P(EDOT-LYZ) with $\overrightarrow{E_{z}}=0.20 \mathrm{kcal} /\left(\mathrm{mol} \cdot \AA \cdot \mathrm{e}^{-}\right): 250 \mathrm{~ns}$ runs; details about the interactions formed by the Glu35 and Asp52 for crystallized LYZ, PEDOT/LYZ with $\overrightarrow{E_{z}}=0.20 \mathrm{kcal} /\left(\mathrm{mol} \cdot \AA \cdot \mathrm{e}^{-}\right)$and P(EDOT-LYZ) with $\overrightarrow{E_{Z}}=0.20 \mathrm{kcal} /\left(\mathrm{mol} \cdot \AA \cdot \AA \cdot \mathrm{e}^{-}\right)$; superposition of the $L Y Z$ crystal structure and the last snapshot from PEDOT/LYZ MD simulation in absence of electric field; and Radial distribution functions (RDF) for the pairs of atoms.

\section{Acknowledgements}

This work was supported by MINECO (MAT2015-69367-R) and AGAUR (2017SGR359). Support for the research of C.A. was received through the prize "ICREA Academia" for excellence in research funded by the Generalitat de Catalunya. J.T. thanks the MINECO for his FPI contract (BES-2013-065536).

Keywords: Adsorption • Conducting polymer • Encapsulation • Molecular dynamics $•$ Protein model

[1] K. Sode, T. Yamazaki, I. Lee, T. Hanashi, W. Tsugawa, Biosens Bioelectron. 2016, 76, 20-28

[2] D. López-Pérez, D. Aradilla, L. J. del Valle, C. Alemán, J. Phys. Chem C 2013, 117, 6607-6619.

[3] a) D. Pankratov, Z. Blum, D. B. Suyatin, V. O. Popov, S. Shleev ChemElectroChem. 2014, 1, 343-346; b) Z. Lv, D. Xie, F. Li, Y. Hu, C. Wei, C. Feng, J. Power Sources 2014, 246, 642-649.

[4] B. Teixeira-Dias, L. J. del Valle, F. Estrany, J. F. Mano, R. L. Reis, C. Alemán, Macromol. Mater. Eng. 2012, 297, 359-368.

[5] T. Hanashi, T. Yamazaki, H. Tanaka, K. Ibebukuro, W. Tsugawa, K. Sode, Sens. Actuator B-Chem. 2014, 196, 429-433.

[6] a) M. Derde, V. Lechevalier, C. Guérin-Dubiard, M.-F. Cochet, S. Jan, F. Baron, M. Gautier, V. Vié, F. Nau, J. Agric. Food Chem. 2013, 61 9922-9929; b) M. Derde, C. Guérin-Dubiard, V. Lechevalier, M.-F. Cochet, S. Jan,F. Baron, M. Gautier, V. Vié, F. Nau, J. Agric. Food Chem. 2014, 62, 1692-1700.

[7] a) K. Liburdi, I. Benucci, F. Palumbo, M. Esti, Food Cont. 2016, 63, 4652; b) M. Uygun, D. Uygun, C. Altunbas, S. Akgol, A. Denizli, Sep. Sci. Technol. 2014, 49, 1270-1278; c) S. I. Park, M. A. Daeschel, Y. Zhao, J. Food Sci. 2004, 69, M215-M221; d) G. Bayramoglu, V. C. Ozalp, M. Yilmaz, U. Guler, B. Salih and M. Y. Arica, Micropor. Mesopor Mat. 2015, 207, 95-104.

[8] a) F. Cavalieri, L. Micheli, S. Kaliappan, B. M. Teo, M. Zhou, G Palleschi, M. Ashokkumar, ACS Appl. Mater. Interfaces 2013, 5, 464 471; b) S. Mahalingan, Z. W. Xu, M. Edirisinghe, Langmuir 2015, 31, 9771-9780; c) A. H. Liao, C. R. Hung, C. F: Lin, Y. C. Lin, H. K. Chen, Sci. Rep. 2017, 7, 41325.

[9] a) M. S. Weiss, G. J. Palm, R. Hilgenfeld, Acta Crystallogr., Sect. D 2000, 56, 952-958; b) L. K. Steinrauf, Acta Crystallogr., Sect. D 1998 , 54, 767-780.

[10] a) C. Lei, P. Wilson, C. Lekakou, J. Power Sources 2011, 196, 7823 7827 ; b) K. S. Ryu, Y. G. Lee, Y. S. Hong,Y.J.Park, X. L. Wu, K. M. Kim, M. G. Kang, N. G. Park, S. H. Chang, Electrochim. Acta 2004, 50, 843-847; c) L. B. Groenendaal, F. Jonas, D. Freitag, H. Pielartzik, J. R. Reynolds, Adv. Mater. 2000, 12, 481-494; d) D. Aradilla, F. Estrany, C. 
Alemán, J. Phys. Chem. C 2011, 115, 8430-8438; e) Q. Wei, M. Mukaida, K. Kirihara, Y. Naitoh, T. Ishida, Materials 2015, 8, 732-750.

[11] J. Poater, J. Casanovas, M. Solà, C. Alemán, J. Phys. Chem. A 2010, 114, 1023-1028.

[12] D. Zanuy, C. Alemán, Soft Matter 2013, 9, 11634-11644.

[13] M. F. McGuire, in Stainless Steels for Design Engineers, (Ed. J. G. Kaufman), ASM International, 2008, Chapter 6, pp. 69-78.

[14] C. Ocampo, R. Oliver, E. Armelin, C. Alemán, F. Estrany, J. Polym. Res. 2006, 13, 193-200.

[15] D. Curcó, D. Zanuy, C. Alemán, J. Comput. Chem. 2003, 24, 12081214.

[16] a) M. Ø. Jensen, D. W. Borhani, K. Lindorff-Larsen, P. Maragakis, V. Jogini, M. P. Eastwood, R. O. Dror, D. E. Shaw, Proc. Natl. Acad. Sci.
USA 2010, 107, 5833-5838; b) J. Su, H. Guo, ACS Nano 2011, 25, 351-359; c) F. Khalili-Araghi, V. Jogini, V. Yarov-Yarovoy, E. Tajkhorshid, B. Roux, K. Schulten, Biophys. J. 2010, 98, 2189-2198.

[17] D. C. Phillips, Sci. Am. 1966, 215, 78-90.

[18] D. E. Koshland, Biol. Rev. 1953, 28, 416-436.

[19] D. J. Vocadlo, G. J. Davies, R. Laine, S. G. Withers, Nature 2001, 412, 835-838.

[20] N. C. Strynadka, M. N. G. James, J. Mol. Biol. 1991, 220, 401-424. 


\section{Entry for the Table of Contents}

\section{ARTICLE}

Atomistic molecular dynamics simulations have been used to examine the influence of lysozyme

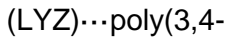
ethylenedioxythiophene) (PEDOT) interactions in the bactericidal activity of LYS/PEDOT biocapacitors when the protein is adsorbed onto the polymer or encapsulated into the polymeric matrix.

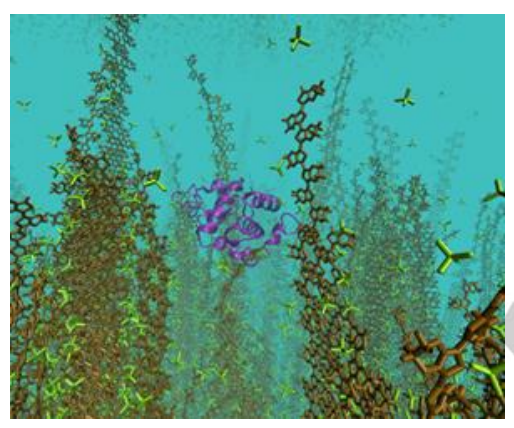

Jordi Triguero, David Zanuy, ${ }^{*}$ and Carlos Alemán*

Page No. - Page No.

Impact of protein-polymer interactions in the antimicrobial activity of lysozyme/poly(3,4ethylenedioxythiophene) biocapacitors 